\title{
Book Review | Deadly Biocultures: The Ethics of Life- Making, by Nadine Ehlers and Shiloh Krupar (University of Minnesota Press, 2019)
}

\author{
Myriam Durocher \\ Carleton University and University of Sydney \\ myriamdurocher@cunet.carleton.ca
}

In this era where violence against Black bodies is denunciated through Black Lives Matter movements, where Indigenous basic human rights are still scorned in colonial contexts, where climate change and its environmental consequences are felt and experienced more harshly by vulnerable populations that have little to do with the rise of temperatures, and where the COVID-19 pandemic is killing the most vulnerable, Deadly Biocultures: The Ethics of Life-Making cruelly astonishes by its relevance. Nadine Ehlers and Shiloh Krupar question the regulatory and "deadly life-making" processes at play in life-making processes and practices in contemporary late Western liberalism, and more specifically as they take form in the US context. As the researchers present it in the very first lines of the book, lifemaking encompasses "all those contemporary efforts to make people live moreto exponentially expand their capacities for life, to optimize and extend what counts as life, and to encourage people to pursue positive life-enhancing practices" (1). While life-making seems like a positive, "ontologically 'good'" (4) quest, the authors unveil how it has deadly consequences for some bodies more than others.

The authors' overarching premise is that life-making cannot be separated from relations of power. Ehlers and Krupar argue that life-making "operates as a regulatory politics of affirmation" (2) in that it "governs by orienting people toward certain ways of looking after themselves, particular goals of health, and 
constrained understandings of body and self" (2). They draw on Foucault's conceptual apparatus of discipline, biopower, and biopolitics to explain the regulatory and normative effects of life-making, but also how life-making leaves room for its twin operation-"letting die"-to emerge. The authors use the expression "deadly life-making" to point out the intricacy of death-making in any life-making processes within the current biopolitical context. They thereby demonstrate the inequalities that permeate make-life processes and show that "not all forms of life are fostered equally" (4). Ehlers and Krupar expose how the life possibilities and life improvements of some are produced at the expense of others. The researchers explore, through the various chapters, three different forms of "letting die" that shape contemporary US biocultures: how life-making practices can "obscure death," "create deadly conditions (that might also be obscured)," and "produce death and/or death effects" (5). Every chapter thus questions the deathly conditions and processes produced by life affirmations that operate in relation to a "particular biomedically framed problem or issue" (7).

Ehlers and Krupar frame "biocultures" as both the arena for investigation and methodology. They approach these biocultures as circumscribed sets of knowledge and practices, associated with particular health-related issues (namely, cancer, racial health, obesity, stem cells, aging, and corpse disposal), that they explore in order to unveil the relations of power at stake. The concept of biocultures itself highlights how biomedicine has become central to everyday life, to our relationships with our selves, bodies, and others: "Biocultures are enmeshed with the biomedical pursuit of fostering biological life-understanding, treating, and preventing disease-and, within biocultures, life is increasingly viewed through the lens of biomedical logics, innovations, and possibilities" (1). Biocultures hence become spaces for questioning how life affirmations operate, regulate, and constrain knowledge and practices in specific health-related issues or terrains, and through various spaces, scales, sites, and actors.

Each chapter of the book examines a key life affirmation that takes form and governs through producing and framing truth/knowledge within these specific biocultures arenas. These life affirmations include to hope (explored within cancer biocultures); to target (racial health); to thrive (obesity and stem cells); to secure (aging); and to green (corpse disposal). Each chapter first presents a life affirmation (e.g., to secure), to then expose how it operates within the biocultures where it takes form (e.g., aging). The analysis that follows includes fieldwork examples and exploration of particular "terrains" that serve to discuss how the particular life affirmation "makes live" (e.g., through techniques, practices, and 
technologies dedicated to secure "aging well," through the biofinancialization of aging populations, through the governance of decline), but also creates deadly conditions (e.g., through deadly care, or neglect of deaths). These terrains, which I did not find explicitly defined within the book, refer to a contained set of knowledge and practices where the life affirmation unfolds. For example, in the chapter dedicated to hope, Ehlers and Krupar explore how this life affirmation operates within public representations of cancer, clinical settings, and personal (and spiritual) encounters. Every chapter concludes with alternative and creative ways of resisting/reworking "the intimate governing operations of affirmation" (13), offering a refreshing and inspiring counter discourse to the dominant one analyzed throughout the chapter. This is arguably a political action in itself as it creates conditions of possibility for disrupting dominant discourses through the very enunciation of these alternatives. Following this same logic, the book ends with a coda, summing up the overarching effects of life-making as the dominant paradigm and arguing for the necessity to question the "let die" functions enacted through it. They stress the need to be attentive to death-making and the death effects of the affirmations of life, as well as the need to develop new forms and practices of care that "counteract dominant affirmations of life and their deadly effects" (163).

The book's innovative analysis consists of thinking about neoliberalism, biomedicalization, and their related practices as intimately entangled with deathmaking. This opens up new ways of caring for bodies, beyond those offered by current dominant biomedical rationalities. The researchers' call for a politics that is attentive to death, especially in the context of biomedicine and life-making, is compelling and offers new ways of thinking (and acting against) the structural and ideological inequalities that mean that "some are made to live (more) at the expense of others" (5). Their ways of approaching these power relations and the issues they raise within specific (bio)cultural realms also add to the developing and growing area of research concerned with biomedicine and biomedicalization processes.

On that note, I was surprised-given the authors' strong and valuable tendency to clearly define and situate within existing literatures the concepts with which they work-to notice the absence of a clear definition of their understanding and use of the term culture in biocultures. While it is made explicit how the concept is tightly associated with biomedicine and its deployment in other realms-social, political, economic, and so on-and how it encompasses a wide heterogeneity of practices, knowledge, actors, scales, sites, and areas of action that contribute to orienting 
one's understanding and action toward their body and life itself at the individual and population level, I could not find a clear definition of how culture is understood in the context of the book. This would have been relevant as it would have provided even more insight into how the researchers conceptualize and approach what they have called "biocultures." Likewise, I wish the authors would have spent a bit more time explaining how their work brings new insights to the body of work concerned with exploring the interconnections or mutual constitutions of "bio" and "culture." The researchers briefly mention that they refuse to distinguish the "bio" from the "cultural," but I wish they had expanded on what that means in the context of their exploration of these biocultures, and how such a concept brings new insights about how "bio" and "culture" mutually inform (or are entangled with) each other in specific ways. Fortunately, while I feel that these elements could have been made more explicit to make an even clearer and stronger case for their proposed notion of "deadly biocultures," it does not impact the overall quality and clarity of the argument the authors develop.

The book is wonderfully written, and proposes a clear, compelling, and provocative argument. Ehlers and Krupar make use of a strong conceptual apparatus, which they take care to define and situate within existing literature. They also effectively situate their work among others who have engaged with analyzing regimes of governance through the lens of the "bio" and from which have emerged concepts such as biomedicalization, biosocialities, biocitizenship, biovalue and biofutures_concepts that are clearly defined and brilliantly integrated into their analysis. Likewise, Ehlers and Krupar clearly distinguish their work from those who are attentive to theorizing life or death at the ontological level, as they pay attention instead to how "death is folded into life through intimate and often mundane forms of governing" (15). Their thorough literature review not only firmly situates their work in the introductory part of the book, but also effectively supports their unfolding argument and analysis in the chapters that follow. I also thoroughly enjoyed reading the endnotes-a quite unusual enjoyable feature, in my opinion-as they were interesting and insightful, effectively completing the discussion in the main text.

I highly recommend this book to people interested in contemporary modes of governance by and through the "bio" as much as those interested in the increasing presence of biomedicine within every realm of the social field. Researchers in areas such as sociology, cultural and biocultural anthropology, and cultural studies should find interest in the authors' perspective on biomedicalization processes, and how they inform the development of new 
cultural norms, body-related knowledge and normativities, and stratified life and death conditions and processes. Likewise, I would recommend the book to those interested in questioning the intersections of culture, life and death, and the politics of life these intersections set into place.

\section{Author Bio}

Myriam Durocher is a postdoctoral fellow at Carleton University (Ottawa, Canada) and at the University of Sydney (Sydney, Australia). In her current postdoctoral research, Myriam explores the temporalities and materialities involved in practices applied to bodies and food materials (such as blood and DNA tests, or pesticides analysis) that aim to prevent health-related risks associated with food ingestion. 